\title{
NGHIÊN CỬU ĐẶC ĐIỂM SIÊU ÂM, TẾ BÀO HỌC CỦA BƯớU NHÂN TUYẾN GIÁP TIRADS 4, TIRADS 5 THEO PHÂN LOẠI EU - TIRADS 2017 Nguyễn Thị Thu ${ }^{1}$, ỗ Trung Quân ${ }^{2}$, Nguyê̂́n Thị Ly $\hat{y}^{3}$ \\ ${ }^{1}$ Bệnh viện Bạch Mai \\ ${ }^{2}$ Truoòng Đại học Y Hà Nội \\ ${ }^{3}$ Trưòng Đại học Y Dược Thái Bình
}

DOI: $10.47122 / v j d e .2020 .42 .9$

\section{ABSTRACT \\ Study on ultrasonic and cytology characteristics of the thyroid neoplasm \\ Tirads 4, Tirads 5 by the EU - Tirads 2017}

Objective: Study on ultrasonic characteristics and cytology characteristics through fine needle aspiration of the thyroid nodules TIRADS 4, TIRADS 5 according to the EU - TIRADS 2017. Methods: Crosssectional descriptive study on 460 patients diagnosed with thyroid nodules on ultrasound, classified TIRADS 4 and / or TIRADS 5 according to the EU - TIRADS 2017 at the Department of Medical Examination and Treatment on request - Bach Mai Hospital. Results: The proportion of patients with a thyroid nodules TIRADS 4 was $64.3 \%$ and TIRADS 5 was $35.7 \%$. The percentage of thyroid nodules with the size from 0.5 to $1 \mathrm{~cm}$ was the highest, accounting for $42.8 \%$. In the TIRADS 5 core group, $26.8 \%$ of the kernel had very negative characteristics; $74.4 \%$ of kernel had irregular banks; $50.6 \%$ of core have microcalcification and $45.7 \%$ of kernel had height characteristics greater than width. In the TIRADS 4 core group: $20.9 \%$ of the kernel has calcification characteristics. The proportion of patients with fine needle aspiration indication was $65.2 \%$. The results according to the Bethesda 2017 classification are: The benign kernels of Bethesda II accounted for the highest rate of $43.7 \%$; Bethesda VI malignant kernels were $35.7 \%$ and the suspected malignancy of Bethesda $\mathrm{V}$ was $12.3 \%$. The rate of carcinoma and suspected carcinoma of the thyroid nodules TIRADS 5 was $75.6 \%$, higher than that of TIRADS 4 was $11.8 \% \quad(p<0.05)$. The proportion of patients with no FNA indication of TIRADS 4 nodules was $48.3 \%$, higher than that of TIRADS $5,10.4 \%(\mathrm{p}<0.05)$. The rate of TIRADS 4 nodules with benign cytology results was $39.9 \%, 14 \%$ higher than that of TIRADS 5 ( $p<0.05)$. Conclusion: Thyroid nodules TIRADS 5 had ultrasound characteristics: $28.6 \%$ was very hypersonic; $74.4 \%$ was the irregular bank; $50.6 \%$ had microcalcification and $45.7 \%$ had height characteristics greater than width. The malignancy rate of TIRADS 5 nodules was statistically significantly higher than that of TIRADS 4 nodules. TIRADS 4 nodules had a higher percentage of no fine needle aspiration indication and a higher rate of benign nodules using fine needle aspiration indication than that of TIRADS 5 .

Keywords: Thyroid nodules, EU - TIRADS, fine needle aspiration (FNA), the Bethesda system.

\section{TÓM TÁT}

Muc tiêu: Nghiên cứu đặc điểm siêu âm và đặc điểm tế bào học qua chọc hút tế bào bằng kim nhỏ của nhân tuyến giáp TIRADS 4 , TIRADS 5 theo phân loại EU - TIRADS 2017. Đối tượng và phương pháp nghiên cúu: Nghiên cứu mô tả cắt ngang trên 460 bệnh nhân được chẩn đoán nhân tuyến giáp trên siêu âm, phân loại TIRADS 4 và / hoặc TIRADS 5 theo phân loại EU - TIRADS 2017 tại khoa Khám chữa bệnh theo yêu cầu - Bệnh viện Bạch Mai. Kết quả: Tỷ lệ bệnh nhân có nhân tuyến giáp TIRADS 4 là $64,3 \%$ và TIRADS 5 là $35,7 \%$. Tỷ lệ nhân tuyến giáp có kích thước từ $0,5-1 \mathrm{~cm}$ là cao nhất chiếm $42,8 \%$. Trong nh óm nh ân TIRADS 5 c ó $26,8 \%$ nhân có đặc điểm rất giảm âm; $74,4 \%$ nhân có bờ không đều; $50,6 \%$ nhân có vi vôi hóa và $45,7 \%$ nhân có đặc điểm chiều cao lớn hơn 
chiều rộng. Trong nhóm nhân TIRADS 4: Có $20,9 \%$ nhân có đặc điểm vôi hóa. Tỷ lệ bệnh nhân có chỉ định chọc hút tế bào bằng kim nhỏ là $65,2 \%$. Kết quả tế học theo phân loại Bethesda 2017 là Nh ân 1 ành $t$ ńh Bethesda II chiếm tỷ lệ cao nhất $43,7 \%$; Nhân ác tính Bethesda VI 1à 35,7\% v à nh ân nghi ngờ ác tính Bethesda $\mathrm{V}$ là $12,3 \%$. Tỷ lệ nhân ác tính và nghi ngờ ác tính của nhân tuyến giáp TIRADS 5 là 75,6\% cao hơn so với TIRADS 4 là $11,8 \%(\mathrm{p}<0,05)$. Tỷ lệ bệnh nhân không có chỉ định FNA của nhân TIRADS 4 là 48,3\% cao hơn so với nhân TIRADS 5 là $10,4 \%$ (p< $0,05)$. Tỷ lệ nhân TIRADS 4 có kết quả tế bào học lành tính là 39,9\% cao hơn so với nhân TIRADS 5 1 à 14\% $(\mathrm{p}<0,05)$. Kết luận: Nh ân TIRADS 5 có đặc điểm siêu âm: $28,6 \%$ rất giảm âm; $74,4 \%$ bờ không đều; $50,6 \%$ có vi vôi hóa và $45,7 \%$ chiều cao lớn hơn chiều rộng. Tỷ lệ ác tính của nhân TIRADS 5 cao hơn có ý nghĩa thống kê so với nhân TIRADS 4. Nhân TIRADS 4 có tỷ lệ không có chỉ định chọc tế bào kim nhỏ và tỷ lệ nhân lành tính khi chọc tế bào kim nhỏ là cao hơn so với nh ân TIRADS 5.

Tù khóa: Bướu nhân tuyến giáp, EU TIRADS, chọc hút tế bào kim nhỏ (FNA), hẹ thống Bethesda.

Chịu trách nhiệm chính: Nguyễn Thị Thu

Ngày nhận bài: 15/8/2020

Ngày phản biện khoa học: 11/9/2020

Ngày duyệt bài: 6/11/2020

Email: thunguyenbvbachmai@gmail.com

\section{1. ĐẠT VẤN Đề}

Bướu nhân tuyến giáp là một bệnh lý khá thường gặp. Bướu nhân tuyến giáp là tình trạng khi có một hoặc nhiều nhân trong nhu mô tuyến giáp, bao gồm nhân lành tính và/ hoặc ác tính. Đa số các bệnh nhân không có biểu hiện lâm sàng và tỷ lệ thăm khám để phát hiện bướu nhân tuyến giáp trên lâm sàng là thấp. Tần suất phát hiện bướu nhân tuyến giáp trên lâm sàng chỉ khoảng 4-7 \% nhưng tr ên si âu âm có thể phát hiện lên tới $19-67 \%$ [6]. Nhiều hiệp hội, nhóm nghiên cứu đã xây dựng các phân loại dựa vào các dấu hiệu siêu âm được đặt tên là TIRADS (Thyroid imaging, Reporting and Data System - Hệ thống dữ liệu hình ảnh và báo cáo tuyến giáp) gi ứp c ác bác sỹ chẩn đoán hình ảnh và lâm sàng có sự thống nhất trong đánh giá tổn thương khu trú tuyến giáp, từ đó đưa ra phương pháp điều trị và theo dõi tốt nhất, trong đó sử dụng bảng phân loại $\mathrm{EU}$ TIRADS 2017 của tác giả Gilles Russ dễ áp dụng, thuận tiện để đánh giá nguy cơ đồng thời có hướng dẫn khuyến cáo chỉ định tiến hành FNA cho bệnh nhân [7].

Việc chẩn đoán tế bào học nhân tuyến giáp là rất quan trọng cho việc điều trị, kế hoạch theo dõi, tiên lượng cho bệnh nhân. Việc chẩn đoán tế bào nhân tuyến giáp đúng sẽ làm giảm đáng kể việc điều trị quá mức bệnh bướu nhân tuyến giáp [8]. Trên thế giới có nhiều đề tài nghiên cứu bướu nhân tuyến giáp dựa trên phân loại TIRADS trên siêu âm đồng thời đánh giá tế bào học nhân tuyến giáp theo phân loại Bethesda 2017. Tuy nhiên, chưa có nghiên cứu nào trên đối tượng các nhân có phân loại nguy cơ cao được phân tích riêng rẽ. Vì vậy chúng tôi tiến hành nghiên cứu đề tài với mục tiêu: Nghiên cứu đặc điểm siêu âm và tế bào học của nhân tuyến giáp TIRADS 4 , TIRADS 5 theo phân loại EU - TIRADS 2017.

\section{2. ĐỐI TƯợNG VÀ PHƯƠNG PHÁP NGHIÊN CÚU}

2.1. Đối tượng nghiên cứu: Gồm 460 người được chẩn đoán là có nhân tuyến giáp trên siêu âm, phân loại TIRADS 4 hoặc TIRADS 5 theo bảng phân loại của EUTIRADS 2017.

\subsection{Phương pháp nghiên cứu}

2.2.1. Thiết kế nghiên cứu: Nghi ên cứu mô tả cắt ngang.

2.2.2. C ách chọn mẫu nghi ên cứu: Mẫu thuận tiện. Những người đến kh ám tại Khoa Kh ám chữa bệnh theo y âu cầu, nếu đủ ti â chuẩn lựa chọn sẽ được lấy $\mathrm{v}$ ào trong nghi ên cứu.

2.2.3. Các bước tiến hành: Các bệnh nhân đến khám bệnh và xét nghiệm, siêu âm theo quy trình và mẫu bệnh án có sẵn. Bao gồm các bước sau:

- Hỏi bệnh và khám lâm sàng. 
- Siêu âm tuyến giáp đánh giá các chỉ số: Số lượng nhân, vị trí nhân, kích thước nhân, độ âm vang nhân, độ phản âm nhân, giới hạn của nhân, dấu hiệu vi vôi hóa, chiều cao so với chiều rộng của nhân. Đối với bướu giáp đa nhân, chúng tôi chọn nhân có phân độ TIRADS cao hơn để mô tả các đặc điểm trên.

- Xét nghiệm FT4, TSH

- Nếu nhân tuyến giáp không có chỉ định FNA $\rightarrow$ Thu thập số liệu.
- Xạ hình tuyến giáp nếu có cường giáp. Đọc kết quả xạ hình.

- Nếu nhân tuyến giáp có chỉ định FNA: Tiến hành chọc hút kim nhỏ dưới hướng dẫn si âu âm (Theo ATA 2015).

- Thu thập kết quả tế bào học theo tiêu chuẩn Bethesda 2017.

- Thu thập kết quả mô bệnh học sau phẫu thuật.

2.4. Phân tích và xử lý số liệu: bằng phần mềm thống kê SPSS 20.0

\section{KÊT QUẢ NGHIÊN CỨU}

Bảng 3.1. Số lượng nh ân tuyến gi áp TIRADS 4 và / hoặc TIRADS 5 ph át hiện tr ân si âu âm

\begin{tabular}{|c|c|c|}
\hline Số lượng nh ân tuyến gi áp & $\mathbf{n}$ & $\mathbf{\%}$ \\
\hline 1 nh ân & 345 & 75,0 \\
\hline Đa nhân & 115 & 25,0 \\
\hline Tống & 460 & 100 \\
\hline
\end{tabular}

Tỷ lệ bệnh nhân đơn nhân chiếm đa số 1 à $75 \%$. Bệnh nh ân có từ 2 nh ân trở 1 ên 1 à $25 \%$.

Bảng 3.2. Tỷ lệ nh ân tuyến gi áp TIRADS 4 và TIRADS 5 trong nh óm nghi ên cứu

\begin{tabular}{|l|c|c|}
\hline \multicolumn{1}{|c|}{ Nh ân tuyến gi áp } & $\mathbf{N}$ & $\mathbf{\%}$ \\
\hline TIRADS 4 & 296 & 64,3 \\
\hline TIRADS 5 & 164 & 35,7 \\
\hline Tồng & 460 & 100 \\
\hline
\end{tabular}

Tỷ lệ bệnh nhân có nhân tuyến giáp TIRADS 4 là $64,3 \%$ và nhân TIRADS 5 là $35,7 \%$.

Bảng 3.3. Đặc điểm kích thước nhân tuyến giáp trên siêu âm

\begin{tabular}{|c|c|c|}
\hline Kích thước & $\mathbf{n}$ & $\mathbf{\%}$ \\
\hline$<0,5 \mathrm{~cm}$ & 85 & 18,5 \\
\hline $0,5-1 \mathrm{~cm}$ & 197 & 42,8 \\
\hline $1-2 \mathrm{~cm}$ & 158 & 34,4 \\
\hline$>2 \mathrm{~cm}$ & 20 & 4,3 \\
\hline Tồn & 460 & 100 \\
\hline
\end{tabular}

Nhân kích thước từ $0,5-1 \mathrm{~cm}$ chiếm tỷ lệ cao nhất là $42,8 \%$. Nhân kích thước $>2 \mathrm{~cm}$ chiếm tỷ lệ thấp nhất là $4,3 \%$.

Bảng 3.4. Đặc điểm các dấu hiệu nghi ngờ ác tính cao trên siêu âm

\begin{tabular}{|c|c|c|c|c|c|c|}
\hline \multirow{2}{*}{\multicolumn{2}{|c|}{ T ńn chất âm $\quad$ TIRADS }} & \multicolumn{2}{|c|}{ TIRADS 4} & \multicolumn{2}{|c|}{ TIRADS 5} & \multirow[t]{2}{*}{ p } \\
\hline & & $\mathrm{n}$ & $\%$ & $\mathbf{n}$ & $\%$ & \\
\hline \multirow{2}{*}{ T ńnh chất âm } & Giảm âm & 296 & 100 & 120 & 73,2 & \multirow{2}{*}{0,000} \\
\hline & Rất giảm âm & 0 & 0 & 44 & 26,8 & \\
\hline \multirow{2}{*}{ Bờ nh ân } & Đều & 296 & 100 & 42 & 25,6 & \multirow{2}{*}{0,000} \\
\hline & Không đều & 0 & 0 & 122 & 74,4 & \\
\hline \multirow{2}{*}{ Vôi hóa } & Kh ông vôi hóa & 234 & 79,1 & 62 & 37,8 & \multirow[b]{2}{*}{0,000} \\
\hline & V ôi hóa & 62 & 20,9 & 19 & 11,6 & \\
\hline
\end{tabular}




\begin{tabular}{|l|l|c|c|c|c|c|}
\hline & Vi vôi hóa & 0 & 0 & 83 & 50,6 & \\
\hline \multirow{2}{*}{$\begin{array}{l}\text { Chiều cao }> \\
\text { chiều rộng }\end{array}$} & Có & 0 & 0 & 89 & 54,3 & \multirow{2}{*}{0,000} \\
\cline { 2 - 6 } & Không & 296 & 100 & 75 & 45,7 & 460 \\
\hline n & & & & & & 46 \\
\hline
\end{tabular}

Trong nhóm nhân TIRADS 5 có $26,8 \%$ nhân có đặc điểm rất giảm âm; 74,4\% nhân có bờ không đều; $50,6 \%$ nhân có vi vôi hóa và $45,7 \%$ nhân có đặc điểm chiều cao lớn hơn chiều rộng. Trong nhóm nhân TIRADS 4: Có 20,9\% nhân có đặc điểm vôi hóa.

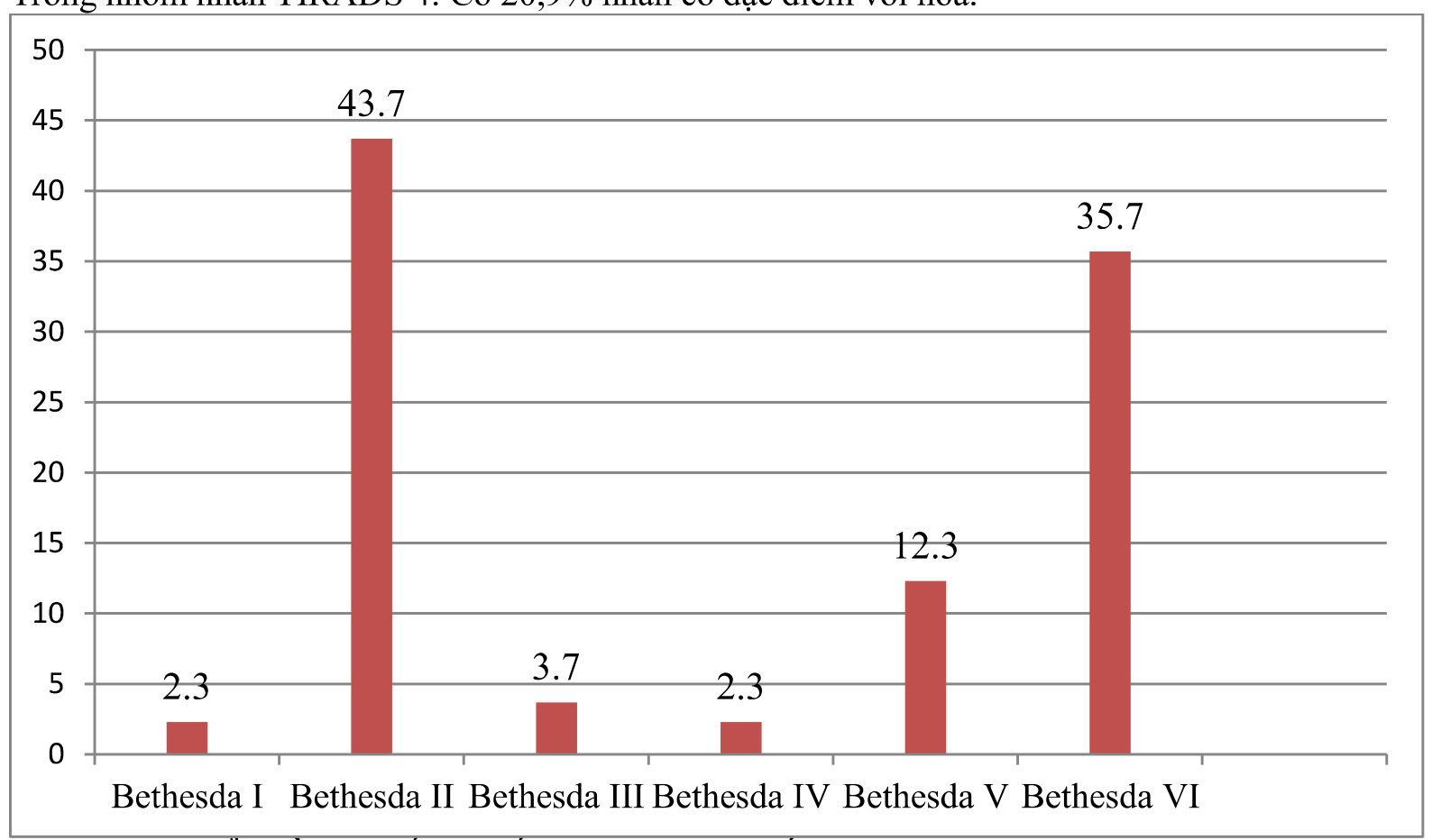

Biểu đồ 3.1. Kết quả tế bào học nhân tuyến giáp TIRADS 4, TIRADS 5

Nh ân 1 ành t ńnh Bethesda II chiếm tỷ lệ cao nhất 43,7\%; Nh ân ác t ńh Bethesda VI 1à 35,7\% và nh ân nghi ngờ ác $t$ ńh Bethesda V 1 à $12,3 \%$.

Bảng 3.5. Đối chiếu ph ân loại TIRADS với FNA v àkết quả tế b ào học

\begin{tabular}{|l|c|c|c|c|c|}
\hline \multirow{2}{*}{ FNA và tế b ào học } & \multicolumn{2}{|c|}{ TIRADS 4 } & \multicolumn{2}{c|}{ TIRADS 5 } & \multirow{2}{*}{ p } \\
\cline { 2 - 5 } & $\mathbf{n}$ & $\mathbf{\%}$ & $\mathbf{n}$ & $\mathbf{\%}$ & \\
\cline { 1 - 5 } Kh ông FNA & 143 & 48,3 & 17 & 10,4 & \\
\hline L ành t ńn & 118 & 39,9 & 23 & 14,0 & \multirow{2}{*}{0,000} \\
\hline Ác t ńnh hoặc nghi ngờ ác t ńh & 35 & 11,8 & 124 & 75,6 & \\
\hline N & 296 & 100 & 164 & 100 & 460 \\
\hline
\end{tabular}

Tỷ lệ bệnh nh ân c ó chỉ định FNA 1 à $65,2 \%$.

Tỷ lệ nh ân ác $t$ ńh vànghi ngờ ác $t$ ńh của nh ân tuyến giáp TIRADS 5 là $75,6 \%$ cao hơn so với TIRADS 4 là 11,8\% (p<0,05).

Tỷ lệ bệnh nh ân kh ông có chỉ định FNA của nhân TIRADS 4 là 48,3\% cao hơn so với nh ân TIRADS 5 là 10,4\% (p<0,05).

Tỷ lệ nh ân TIRADS 4 c ó kết quả tế b ào học lành tính là 39,9\% cao hơn so với nh ân TIRADS 51 à $14 \%(\mathrm{p}<0,05)$. 


\section{BÀn LUẦN}

Trong nghiên cứu của chúng tôi, tỷ lệ bướu giáp đơn nhân TIRADS 4 hoặc TIRADS 5 phát hiện trên siêu âm là $75 \%$ và đa nhân là $25 \%$. Theo nghiên cứu của tác giả Warinthorn tỷ lệ bướu đơn nhân là $51,6 \%$ và bướu đa nhân là 48,2\% [9]. Có sự khác biệt là do nghiên cứu của chúng tôi trên nhân tuyến giáp TIRADS 4, TIRADS 5 nên không phải là tất cả nhân tuyến giáp phát hiện trên siêu âm.

Tỷ lệ bệnh nhân có nhân tuyến giáp TIRADS 41 à $64,3 \%$ và nh ân TIRADS 51 à $35,7 \%$. Theo nghiên cứu của tác giả Trần Thúy Hồng khi sử dụng bảng phân loại TIRADS của tác giả Jin Joung Kwak và cs (2011) cho kết quả: tỷ lệ nhân tuyến giáp TIRADS $4 \mathrm{a}, 4 \mathrm{~b}$ và $4 \mathrm{c} 1$ à $55,5 \%$ và nh ân TIRADS 5 là 2,8\% [1]. Có sự khác biệt nhu vậy là do tác giả nghiên cứu tất cả các nhân tuyến giáp và sử dụng phân loại TIRADS 2011.

Đặc điểm kích thước nhân tuyến giáp: Trong nghiên cứu của chúng tôi, nhân có kích thước từ $0,5-1 \mathrm{~cm}$ chiếm tỷ lệ cao nhất $42,8 \%$. Kết quả của chúng tôi tương tự như kết quả của tác giả Đào Văn Luận nhân có kích thước từ $0,6-1 \mathrm{~cm}$ chiếm tỷ lệ cao nhất $37 \%$ [2]. Kết quả của chúng tôi khác với kết quả nghiên cứu của Nguyễn Thị Hải Yến, nhân có kích thước $1-2 \mathrm{~cm}$ chiếm tỷ lệ cao nhất $42 \%$ [5]. Có sự khác biệt là do nghiên cứu của chúng tôi trên nhân có nguy cơ ác tính cao hơn so với nghiên cứu trên toàn bộ nhân tuyến giáp. Điều này cho thấy, đánh giá đúng TIRADS của nhân tuyến giáp, sẽ cho chỉ định chọc hút tế bào kim nhỏ đúng và phát hiện nhiều nhân mặc dù có kích thước nhỏ nhưng có thể đã là nhân ác tính. Từ đó có chỉ định điều trị đúng với những nhân có kích thước nhỏ, thậm chí $<0,5 \mathrm{~cm}$.

Theo phân loại EU - TIRADS 2017, chỉ cần có một trong ác dấu hiệu nghi ngờ ác tính cao (nhân rất giảm âm, bờ không đều, vi vôi hóa, chiều cao lớn hơn chiều rộng) sẽ phân loại thành nhân TIRADS 5. Tỷ lệ nhân TIRADS 5 rất giảm âm trong nghiên cứu của chúng tôi 1 à $26,8 \%$. Trong nghiên cứu của chúng tôi có 122 bệnh nhân có đặc điểm nhân bờ không đều chiếm tỷ lệ 74,4\%. Vi vôi hóa là một trong những dấu hiệu có độ nhạy cao nhất trong chẩn đoán ung thư tuyến giáp từ 85 $-95 \%$, giá trị dự báo dương tính là 41,8 $94,2 \%$ [10]. Nghiên cứu trên 460 BN chúng tôi thu được kết quả: Nhân vi vôi hóa chiếm tỷ lệ $50,6 \%$; nhân vôi hóa là $11,6 \%$ và nhân không vôi hóa là $37,8 \%$. Kết quả của chúng tôi khá tương đồng với một số kết quả của các tác giả trong nước [2], [5]. Có 89 bệnh nhân trong nhóm nhân TIRADS 5 có đặc điểm chiều cao lớn hơn chiều rộng chiếm tỷ lệ $54,3 \%$. Sự phát triển của ung thư giáp cũng giống như sự phát triển của ung thư vú, các bướu ác tính thường phát triển xuyên qua mặt phẳng của mô bình thường, trong khi các bướu lành tính thường phát triển song song với mặt phẳng của mô bình thường. Do vậy các bướu ác tính thường có hình dạng chiều cao lớn hơn chiều rộng [11].

Trong nghiên cứu của chúng tôi có 300 bệnh nhân được chọc hút tế bào kim nhỏ, thu được kết quả: Nhân lành tính Bethesda II chiếm tỷ lệ cao nhất $43,7 \%$; Nhân ác tính Bethesda VI là $35,7 \%$ và nhân nghi ngờ ác tính Bethesda V là $12,3 \%$. Như vậy tỷ lệ nhân ác tính hoặc nghi ngờ ác tính là $48 \%$. Kết quả nghiên cứu của chúng tôi tương tự như kết quả của tác giả Vũ Bích Nga trên 106 bệnh nhân được chọc hút tế bào kim nhỏ cho kết quả: $50 \%$ bướu nhân lành tính; 24,5 nhân ác tính và $20,8 \%$ nhân nghi ngờ ác tính [3]. Kết quả của chúng tôi khác với kết quả nghiên cứu của tác giả Đặng Bích Ngọc: Tỷ lệ nhân lành tính là $86 \%$; nghi ngờ ác tính $4 \%$, nhân ác tính $7,3 \%$ và phiến đồ không thỏa đáng là $2,7 \%$ [4]. Có sự khác biệt như vậy có lẽ là do đối tượng trong nghiên cứu của chúng tôi là nhân TIRADS 4, TIRADS 5 là những nhân có nguy cơ ác tính cao hơn. Đồng thời chúng tôi tiến hành FNA cho những nhân kích thước nhỏ $<0,5 \mathrm{~cm}$ nên tỷ lệ phát hiện nhân ác tính hoặc nghi ngờ ác tính cũng cao hơn so với các nghiên cứu trước đó.

\section{KẾT LUẬN}

Qua khảo sát 460 bệnh nhân có nhân tuyến gi áp TIRADS 4 và / hoặc TIRADS 5 theo 
phân loại EU - TIRADS 2017 chúng tôi thu được kết quả sau:

- Tỷ lệ nhân tuyến giáp TIRADS 4 là 64,3\% vành ân TIRADS 51 à 35,7\%.

- Nhân kích thước từ $0,5-1 \mathrm{~cm}$ chiếm tỷ lệ cao nhất là $42,8 \%$.

- Trong nhóm nh ân TIRADS 5 c ó $26,8 \%$ nh ân c ó đặc điểm rất giảm âm; $74,4 \%$ nhân có bờ không đều; $50,6 \%$ nhân có vi vôi hóa và $45,7 \%$ nhân có đặc điểm chiều cao lớn hơn chiều rộng. Trong nhóm nhân TIRADS 4: Có $20,9 \%$ nhân có đặc điểm vôi hóa.

- Kết quả tế b ào học trên 300 nhân được FNA: Nh ân 1 ành $\mathrm{t}$ ńh Bethesda II chiếm tỷ lệ cao nhất 43,7\%; Nh ân ác t ńh Bethesda VI 1 à $35,7 \%$ và nh ân nghi ngờ ác $t$ ńh Bethesda $\mathrm{V}$ 1 à $12,3 \%$. $65,2 \%$

- Tỷ lệ bệnh nhân có chỉ định FNA là

- Tỷ lệ nhân ác t ńh và nghi ngờ ác t ńh của nh ân tuyến gi áp TIRADS 51 à 75,6\% cao hơn so với TIRADS 41 à $11,8 \%(\mathrm{p}<0,05)$.

- Tỷ lệ bệnh nh ân kh ông có chỉ định FNA của nhân TIRADS 4 là $48,3 \%$ cao hơn so với nh ân TIRADS 51 à 10,4\% $(\mathrm{p}<0,05)$.

- Tỷ lệ nhân TIRADS 4 có kết quả tế b ào học lành tính là $39,9 \%$ cao hơn so với nh ân TIRADS 5 là $14 \%(\mathrm{p}<0,05)$.

\section{TÀI LIÊU THAM KHẢO}

1. Trần Thúy Hồng, Bùi Văn Lệnh (2013). Đặc điểm hình ảnh và giá trị của siêu âm trong chẩn đoán các tổn thương khu trú tuyến giáp. Luận văn thạc sĩ y hoc. Trường Đại học Y Hà Nội.

2. Nguyễn Văn Luận, Phạm Tuấn Cảnh (2017). Đánh giá vai trò TIRADS, chọc hút kim nhỏ và sinh thiết tức thì trong chẩn đoán và điều trị bướu nhân tuyến gi áp. Luận văn thạc sĩ y học. Trường Đại học Y Hà Nội.

3. Vũ Bích Nga (2012). Đặc điểm bướu nhân tuyến giáp qua chọc hút tế bào bằng kim nhỏ dưới hướng dẫn siêu âm. Tạp ch íyhoc thục hành.
4. Đặng Bích Ngọc, Nguyễn Khoa Diệu Vân, Phạm Thu Hà (2015). Vai trò của siêu âm và chọc hút kim nhỏ dưới hướng dẫn của siêu âm trong chẩn đoán ung thư tuyến giáp. Tạp chí nghiên cứu y hoc, 97 (5), $115-122,2015$.

5. Nguyễn Thị Hải Yến, Vũ Bích Nga (2016). Nghiên cứu đặc điểm lâm sàng và cận lâm sàng bệnh bướu nhân tuyến gi áp. Luận văn bác sĩ chuyên khoa cấp II. Trường Đại học Y Hà Nội.

6. Tan, G.H. and H. Gharib, Thyroid incidentalomas: management approaches to nonpalpable nodules discovered incidentally on thyroid imaging. Ann Intern Med, 1997. 126(3): p. 226-31.

7. Russ, G., et al., European Thyroid Association Guidelines for Ultrasound Malignancy Risk Stratification of Thyroid Nodules in Adults: The EU-TIRADS. Eur Thyroid J, 2017. 6(5): p. 225-237.

8. Haugen, B.R., et al., 2015 American Thyroid Association Management Guidelines for Adult Patients with Thyroid Nodules and Differentiated Thyroid Cancer: The American Thyroid Association Guidelines Task Force on Thyroid Nodules and Differentiated Thyroid Cancer. Thyroid, 2016. 26(1): p. 1-133.

9. Phuttharak, W., C. Somboonporn, and G. Hongdomnern, Diagnostic performance of gray-scale versus combined gray-scale with colour doppler ultrasonography in the diagnosis of malignancy in thyroid nodules. Asian Pac J Cancer Prev, 2009. 10(5): p. 759-64.

10. Frates, M.C., et al., Management of thyroid nodules detected at US: Society of Radiologists in Ultrasound consensus conference statement. Radiology, 2005. 237(3): p. 794-800.

11. Khati, N., et al., Ultrasound of the thyroid and parathyroid glands. Ultrasound Q, 2003. 19(4): p. 162-76. 